\title{
SOME NEW RESULTS ASSOCIATED WITH THE GENERALIZED LOMMEL-WRIGHT FUNCTION
}

\author{
S. HAQ, K.S. NisAR, A.H. KHAN
}

ABstract. The aim of this article is to establish a new class of unified integrals associated with the generalized Lommel-Wright functions.Some integrals involving trigonometric, generalized Bessel and Struve functions are also mentioned as special cases of the main results. Further, we establish two reduction formulas for the Wright hypergeometric function.

2010 Mathematics Subject Classification: 33B20, 33C20, 33B15.

Keywords: Gamma function, generalized Wright hypergeometric function ${ }_{p} \psi_{q}$, Hypergeometric function ${ }_{p} F_{q}$, generalized Lommel-Wright functions $J_{\nu, \lambda}^{\mu m}(z)$, LavoieTrottier integral formula.

\section{INTRODUCTION}

The Wright hypergeometric function defined by the series [15]:

$$
{ }_{p} \psi_{q}\left[\begin{array}{c}
\left(\alpha_{1}, A_{1}\right), . .,\left(\alpha_{p}, A_{p}\right) ; \\
\left(\beta_{1}, B_{1}\right) \ldots,\left(\beta_{q}, B_{q}\right)
\end{array}\right]=\sum_{k=0}^{\infty} \frac{\prod_{j=1}^{p} \Gamma\left(\alpha_{j}+A_{j} k\right) z^{k}}{\prod_{j=1}^{q} \Gamma\left(\beta_{j}+B_{j} k\right) k !},
$$

where the coefficients $A_{1}, \ldots . A_{p}$ and $B_{1}, \ldots . B_{q}$ are positive real numbers such that

$$
1+\sum_{j=1}^{q} B_{j}-\sum_{j=1}^{p} A_{j} \geq 0 .
$$

can be slightly generalized (1) as given below.

$$
{ }_{p} \psi_{q}\left[\begin{array}{c}
\left(\alpha_{1}, 1\right), . .,\left(\alpha_{p}, 1\right) ; \\
\left(\beta_{1}, 1\right) \ldots,\left(\beta_{q}, 1\right)
\end{array}\right]=\frac{\prod_{j=1}^{p} \Gamma\left(\alpha_{j}\right)}{\prod_{j=1}^{q} \Gamma\left(\beta_{j}\right)}{ }_{p} F_{q}\left[\begin{array}{c}
\alpha_{1}, . ., \alpha_{p} ; \\
\beta_{1}, \ldots, \beta_{q}
\end{array}\right]
$$


where ${ }_{p} F_{q}$ is the generalized hypergeometric function defined by $[15,13]$

$$
{ }_{p} F_{q}\left[\begin{array}{c}
\alpha_{1}, . ., \alpha_{p} ; \\
\beta_{1}, \ldots, \beta_{q}
\end{array}\right]=\sum_{k=0}^{\infty} \frac{\left(\alpha_{1}\right)_{n}, \ldots .,\left(\alpha_{p}\right)_{n} z^{n}}{\left(\beta_{1}\right)_{n}, \ldots .,\left(\beta_{q}\right)_{n} n !}={ }_{p} F_{q}\left(\alpha_{1}, \ldots, \alpha_{p} ; \beta_{1}, \ldots, \beta_{q} ; z\right),
$$

where $(\vartheta)_{n}$ is denote the Pochhammer symbol [15].

The series representation of the generalized Lommel Wright function as [5];

$$
\begin{array}{r}
J_{\nu, \lambda}^{\mu, m}(z)=\sum_{k=0}^{\infty} \frac{(-1)^{k} \Gamma(k+1)\left(\frac{z}{2}\right)^{2 k+\nu+2 \lambda}}{\Gamma(\lambda+k+1)^{m} \Gamma(\nu+k \mu+\lambda+1) k !}, \\
(z \in \mathbb{N} /(-\infty, 0] m \in \mathbb{N}, \nu, \lambda \in \mathbb{C}, \mu>0) .
\end{array}
$$

Also, we have the following relations of generalized Lommel Wright functions with trigonometric functions and the generalized Bessel function and Struve function:

$$
\begin{gathered}
\left.J_{1 / 2,0}^{1,1}(z)=\sqrt{(} \frac{2}{\pi z}\right) \sin (z) \\
\left.J_{-1 / 2,0}^{1,1}(z)=\sqrt{(} \frac{2}{\pi z}\right) \cos (z) \\
J_{\nu, \lambda}^{\mu, 1}(z)=\mathbb{J}_{\nu, \lambda}^{\mu}(z) \\
J_{\nu, 1 / 2}^{1,1}(z)=H_{\nu}(z) .
\end{gathered}
$$

Here, we recall the following Lavoie and Trottier integral formula[8].

$$
\int_{0}^{1} t^{\kappa-1}(1-t)^{2 \vartheta-1}\left(1-\frac{t}{3}\right)^{2 \kappa-1}\left(1-\frac{t}{4}\right)^{\vartheta-1} d t=\left(\frac{2}{3}\right)^{2 \kappa} \frac{\Gamma(\kappa) \Gamma(\vartheta)}{\Gamma(\kappa+\vartheta)}
$$

where $\Re(\kappa)>0$ and $\Re(\vartheta)>0$.

Various generalizations and cases of Lommel-Wright function have been investigated (see, for details, $[1,14,7]$ ). For more details of integral involving various special functions, one may be referred to the recent research papers $[4,9,10,11]$

Integral formulas involving Lommel-Wright functions have been developed by many authors (see, e.g., $[2,3,6,12]$ ). In this sequel, here, we aim at establishing certain new generalized integral formula involving the generalized Lommel-Wright function $J_{\nu, \lambda}^{\mu, m}(z)$ interesting integral formulas which are derived as special cases. 


\section{Main Results}

The integrals involving Lommel-Wright function is given in this section.

Theorem 1. For $\eta, \theta \in \mathbb{C}$ and $t>0$ with $\Re(\eta)>0, \Re(\theta)>0$, the following integral formula holds true

$$
\begin{aligned}
& \int_{0}^{1} x^{\eta-1}(1-x)^{2 \theta-1}\left(1-\frac{x}{3}\right)^{2 \eta-1}\left(1-\frac{x}{4}\right)^{\theta-1} J_{\nu, \lambda}^{\mu, m}\left(y\left(1-\frac{t}{4}\right)(1-t)^{2}\right) d t \\
& =\Gamma(\eta)\left(\frac{2}{3}\right)^{2 \eta}\left(\frac{y}{2}\right)^{\nu+2 \lambda} \\
& \times{ }_{2} \psi_{m+2}\left[(\lambda+1,1), \ldots,(\lambda+1,1),(\eta+\theta+\nu+2 \lambda, 2),(\nu+\lambda+1, \mu) ;-\frac{y^{2}}{4}\right] .
\end{aligned}
$$

Proof. On using (5) in the integrand of (11) and then interchanging the order of integral sign and summation which is verified under the given conditions, we get

$$
\begin{aligned}
& \int_{0}^{1} t^{\eta-1}(1-t)^{2 \theta-1}\left(1-\frac{t}{3}\right)^{2 \eta-1}\left(1-\frac{t}{4}\right)^{\theta-1} J_{\nu, \lambda}^{\mu, m}\left(y\left(1-\frac{t}{4}\right)(1-t)^{2}\right) d t \\
& =\left(\frac{y}{2}\right)^{\nu+2 \lambda} \sum_{k=0}^{\infty} \frac{\Gamma(k+1)\left(-y^{2} / 4\right)^{k}}{\Gamma(\lambda+k+1)^{m} \Gamma(\nu+k \mu+\lambda+1) k !} \\
& \times \int_{0}^{1} t^{\eta-1}(1-t)^{2(\theta+2 k+\nu+2 \lambda)-1}\left(1-\frac{t}{3}\right)^{2 \eta-1}\left(1-\frac{t}{4}\right)^{(\theta+2 k+\nu+2 \lambda)-1} d t
\end{aligned}
$$

Now using (10) in the above equation we get

$$
\begin{aligned}
& \int_{0}^{1} t^{\eta-1}(1-t)^{2 \theta-1}\left(1-\frac{t}{3}\right)^{2 \eta-1}\left(1-\frac{t}{4}\right)^{\theta-1} J_{\nu, \lambda}^{\mu, m}\left(y\left(1-\frac{t}{4}\right)(1-t)^{2}\right) d t=\left(\frac{2}{3}\right)^{2 \eta} \\
& \times \Gamma(\eta)\left(\frac{y}{2}\right)^{\nu+2 \lambda} \sum_{k=0}^{\infty} \frac{\Gamma(k+1) \Gamma(\theta+\nu+2 \lambda+2 k)\left(\frac{-y^{2}}{4}\right)^{k}}{\Gamma(\lambda+k+1)^{m} \Gamma(\eta+\theta+\nu+2 \lambda+2 k) \Gamma(\nu+k \mu+\lambda+1) k !} .
\end{aligned}
$$

Finally, using (1) in the above equation, we get our assertion (11).

Theorem 2. The following integral formula holds true: For $\eta, \theta \in C$ and $t>0$ with 


$$
\begin{aligned}
& \Re(\theta)>0, \Re(\eta)>0, \\
& \quad \int_{0}^{1} t^{\eta-1}(1-t)^{2 \theta-1}\left(1-\frac{t}{3}\right)^{2 \eta-1}\left(1-\frac{t}{4}\right)^{\theta-1} J_{\nu, \lambda}^{\mu, m}\left(t y\left(1-\frac{t}{3}\right)^{2}\right) d t \\
& \quad=\left(\frac{2}{3}\right)^{2(\eta+\nu+2 \lambda)}\left(\frac{y}{2}\right)^{\nu+2 \lambda} \Gamma(\theta) \\
& \quad \times{ }_{2} \psi_{m+2}\left[\begin{array}{cc}
(1,1),(\eta+\nu+2 \lambda, 2) ; & \frac{-4 y^{2}}{81}
\end{array}\right] .
\end{aligned}
$$

Proof. On using (5) in the integrand of (14) and then interchanging the order of integral sign and summation which is verified under the given conditions, we get

$$
\begin{aligned}
& \int_{0}^{1} t^{\eta-1}(1-t)^{2 \theta-1}\left(1-\frac{t}{3}\right)^{2 \eta-1}\left(1-\frac{t}{4}\right)^{\theta-1} J_{\nu, \lambda}^{\mu, m}\left(t y\left(1-\frac{t}{3}\right)^{2}\right) d t \\
& =(y / 2)^{\nu+2 \lambda} \sum_{k=0}^{\infty} \frac{\Gamma(k+1)\left(-\frac{y^{2}}{4}\right)^{k}}{\Gamma(\lambda+k+1)^{m} \Gamma(\nu+k \mu+\lambda+1) k !} \\
& \times \int_{0}^{1} t^{\eta+\nu+2 \lambda+2 k-1}(1-t)^{2 \theta-1}\left(1-\frac{t}{3}\right)^{2(\eta+\nu+2 \lambda+2 k)-1}\left(1-\frac{t}{4}\right)^{\theta-1} d t .
\end{aligned}
$$

Now using (10) in the above equation we get

$$
\begin{aligned}
& \int_{0}^{1} t^{\eta-1}(1-t)^{2 \theta-1}\left(1-\frac{t}{3}\right)^{2 \eta-1}\left(1-\frac{t}{4}\right)^{\theta-1} J_{\nu, \lambda}^{\mu, m}\left(y\left(1-\frac{t}{4}\right)\right) d t \\
& =\left(\frac{2}{3}\right)^{2(\eta+\nu+2 \lambda)} \Gamma(\theta)\left(\frac{y}{2}\right)^{\nu+2 \lambda} \\
& \times \sum_{k=0}^{\infty} \frac{\Gamma(k+1) \Gamma(\eta+\nu+2 \lambda+2 k)\left(\frac{-4 y^{2}}{81}\right)^{k}}{\Gamma(\lambda+k+1)^{m} \Gamma(\nu+k \mu+\lambda+1) \Gamma(\eta+\theta+\nu+2 \lambda+2 k) k !} .
\end{aligned}
$$

Finally, using (1) in the above equation, we get our assertion (14).

Next we consider other variations of Theorem 1 and Theorem 2 in the form of corollaries. 
Corollary 3. In (13), on separating the hypergeometric series into its even and odd terms, we get,

$$
\begin{aligned}
& \int_{0}^{1} t^{\eta-1}(1-t)^{2 \theta-1}\left(1-\frac{t}{3}\right)^{2 \eta-1}\left(1-\frac{t}{4}\right)^{\theta-1} J_{\nu, \lambda}^{\mu, m}\left(y\left(1-\frac{t}{4}\right)(1-t)^{2}\right) d t=\left(\frac{2}{3}\right)^{2 \eta} \sqrt{\pi} \\
& \times\left(\frac{y}{2}\right)^{\nu+2 \lambda} \Gamma(\eta)_{2} \psi_{m+3}\left[\begin{array}{cc}
(1,2),(\theta+\nu+2 \lambda, 4) ; & \frac{y^{4}}{64}
\end{array}\right] \\
& -\left(\frac{2}{3}\right)^{2 \eta} \frac{\sqrt{\pi}}{2}\left(\frac{y}{2}\right)^{\nu+2 \lambda+2} \Gamma(\eta)
\end{aligned}
$$

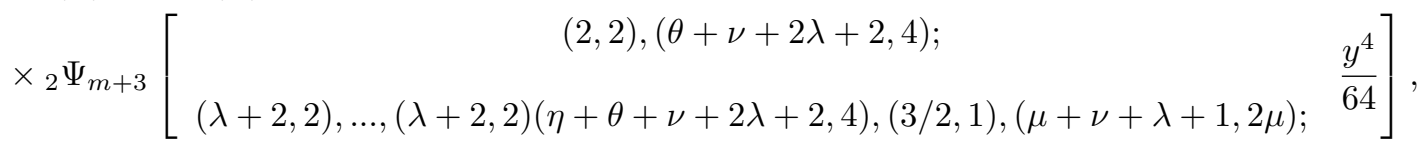

where $\Re(\eta)>0$ and $\Re(\theta)>0$.

Corollary 4. On expanding the r.h.s of (16) in series form and then separating the resulting series into its even and odd terms, we obtain.

$$
\begin{aligned}
& \int_{0}^{1} t^{\eta-1}(1-t)^{2 \theta-1}\left(1-\frac{t}{3}\right)^{2 \eta-1}\left(1-\frac{t}{4}\right)^{\theta-1} J_{\nu, \lambda}^{\mu, m}\left(t y\left(1-\frac{t}{3}\right)^{2}\right) d t \\
& =\left(\frac{2}{3}\right)^{2(\eta+\theta+2 \lambda)} \sqrt{\pi}\left(\frac{y}{2}\right)^{\nu+2 \lambda} \Gamma(\theta) \\
& \times{ }_{2} \psi_{m+3}\left[\begin{array}{cc}
(1,2),(\eta+\theta+2 \lambda, 4) ; \\
(\lambda+1,2), \ldots,(\lambda+1,2)(1 / 2,1),(\eta+\theta+\nu+2 \lambda, 4),(\nu+\lambda+1,2 \mu) ;
\end{array}\right] \\
& +\left(\frac{2}{3}\right)^{2(\eta+\theta+2 \lambda)} \frac{y^{4}}{2}\left(-\frac{4 y^{2}}{81}\right)\left(\frac{y}{2}\right)^{\nu+2 \lambda} \Gamma(\theta) \\
& \times{ }_{2} \Psi_{m+3}\left[\begin{array}{c}
(\lambda, 2),(\eta+\theta+2 \lambda+2,4) ; \\
(\lambda+2,2), \ldots,(\lambda+2,2)(3 / 2,1),(\eta+\theta+\nu+2 \lambda+2,4),(\nu+\lambda+\mu+1,2 \mu) ;
\end{array}\right]
\end{aligned}
$$

where $\Re(\eta)>0$ and $\Re(\theta)>0$.

\section{Special Cases}

In this section, we derive some integrals containing trigonometric function and generalized Lommel-Wright function as follows: 
Corollary 5. If we take $m=1, \mu=1, \lambda=0$ and $\nu=1 / 2$ in (11) and then by using (6), we derive the following integral formula:

$$
\begin{array}{r}
\int_{0}^{1} t^{\eta-1}(1-t)^{2(\theta-1 / 2)-1}\left(1-\frac{t}{3}\right)^{2 \eta-1}\left(1-\frac{t}{4}\right)^{(\theta-1 / 2)-1} \sin \left(y(1-t / 4)(1-t)^{2}\right) d t \\
=\left(\frac{2}{3}\right)^{2 \eta} \sqrt{\pi}\left(\frac{y}{2}\right) \Gamma(\eta)_{1} \psi_{2}\left[\begin{array}{cc}
(\theta+1 / 2,2) ; & y^{2} \\
(\eta+\theta+1 / 2,2),\left(\frac{3}{2}, 1\right) ; & -\frac{1}{4}
\end{array}\right]
\end{array}
$$

where $\Re(\eta)>0$ and $\Re(\theta)>0$.

Corollary 6. Again by taking $m=1, \mu=1, \lambda=0$ and $\nu=1 / 2$ in (14) and then by using (6), we deduce the following integral formula:

$$
\begin{aligned}
\int_{0}^{1} t^{\left(\eta-\frac{1}{2}\right)-1}(1-t)^{2 \theta-1}\left(1-\frac{t}{3}\right)^{2\left(\eta-\frac{1}{2}\right)-1} & \left(1-\frac{t}{4}\right)^{\theta-1} \sin \left(t y\left(1-\frac{t}{3}\right)^{2}\right) d t \\
=\left(\frac{2}{3}\right)^{2(\eta+\theta)} \sqrt{\pi}\left(\frac{y}{2}\right) \Gamma(\theta)_{1} \psi_{2} & {\left[\begin{array}{cc}
(\eta+\theta, 2) ; & 4 y^{2} \\
\left(\eta+\theta+\frac{1}{2}, 2\right),\left(\frac{3}{2}, 1\right) ; & -\frac{4}{81}
\end{array}\right] }
\end{aligned}
$$

where $\Re(\eta)>0$ and $\Re(\theta)>0$.

Corollary 7. If we put $m=1, \mu=1, \lambda=0$ and $\nu=1 / 2$ in (17) and then by using (6), we obtain:

$$
\begin{aligned}
& \int_{0}^{1} t^{\eta-1}(1-t)^{2\left(\theta-\frac{1}{2}\right)-1}\left(1-\frac{t}{3}\right)^{2 \eta-1}\left(1-\frac{t}{4}\right)^{\left(\theta-\frac{1}{2}\right)-1} \sin \left(y\left(1-\frac{t}{4}\right)(1-t)^{2}\right) d t \\
& =\left(\frac{2}{3}\right)^{2 \eta}\left(\frac{y \pi}{2}\right) \Gamma(\eta)_{1} \psi_{3}\left[\begin{array}{cc}
\left(\theta+\frac{1}{2}, 4\right) ; \\
\left(\eta+\theta+\frac{1}{2}, 4\right),\left(\frac{3}{2}, 1\right),(1 / 2,1) ;
\end{array}\right] \\
& -\left(\frac{2}{3}\right)^{2 \eta}\left(\frac{y^{3} \pi}{16}\right) \Gamma(\eta)_{1} \psi_{3}\left[\begin{array}{cc}
\left(\theta+\frac{5}{2}, 4\right) ; \\
\left(\eta+\theta+\frac{5}{2}, 4\right),\left(\frac{3}{2}, 1\right),\left(\frac{5}{2}, 2\right) ;
\end{array}\right],
\end{aligned}
$$

where $\Re(\eta)>0$ and $\Re(\theta)>0$.

Corollary 8. Further we take $m=1, \mu=1, \lambda=0$ and $\nu=1 / 2$ in (18) and then by 
using (6), we obtain:

$$
\begin{aligned}
\int_{0}^{1} t^{\left(\eta-\frac{1}{2}\right)-1}(1-t)^{2 \theta-1}\left(1-\frac{t}{3}\right)^{2\left(\eta-\frac{1}{2}\right)-1}\left(1-\frac{t}{4}\right)^{\theta-1} \sin \left(t y\left(1-\frac{t}{3}\right)^{2}\right) d t \\
=\left(\frac{2}{3}\right)^{2(\eta+\theta)}\left(\frac{\pi y}{2}\right) \Gamma(\theta)_{1} \psi_{3}\left[\begin{array}{cc}
(\eta+\theta, 4) ; & 4 y^{4} \\
\left(\eta+\theta+\frac{1}{2}, 4\right),\left(\frac{1}{2}, 1\right),\left(\frac{3}{2}, 2\right) ; & \left.\frac{6561}{(\eta+1}\right]
\end{array}\right] \\
+\left(\frac{2}{3}\right)^{2(\eta+\theta)} \Gamma(\theta)\left(\frac{4 y^{3}}{81}\right)_{1} \psi_{3}\left[\begin{array}{cc}
(\eta+\theta+2,4) ; & \frac{4 y^{4}}{6561}
\end{array}\right],
\end{aligned}
$$

where $\Re(\eta)>0$ and $\Re(\theta)>0$.

Corollary 9. If we take $m=1, \mu=1, \lambda=0$ and $\nu=-1 / 2$ in (11)and then by using (7), we get the following integral formula:

$$
\begin{array}{r}
\int_{0}^{1} t^{\eta-1}(1-t)^{2\left(\theta-\frac{1}{2}\right)-1}\left(1-\frac{t}{3}\right)^{2 \eta-1}\left(1-\frac{t}{4}\right)^{\left(\theta-\frac{1}{2}\right)-1} \cos \left(y\left(1-\frac{t}{4}\right)\left(1-t^{2}\right) d t\right. \\
=\left(\frac{2}{3}\right)^{2 \eta} \sqrt{\pi} \Gamma(\eta)_{1} \psi_{2}\left[\begin{array}{cc}
\left(\theta-\frac{1}{2}, 2\right) ; & -\frac{y^{2}}{4} \\
\left(\eta+\theta-\frac{1}{2}, 2\right),\left(\frac{1}{2}, 1\right) ; &
\end{array}\right],
\end{array}
$$

where $\Re(\eta)>0$ and $\Re(\theta)>0$.

Corollary 10. Again we take $m=1, \mu=1, \lambda=0$ and $\nu=-1 / 2$ in (14) and then by using (7), we get the following integral formula:

$$
\begin{aligned}
\int_{0}^{1} t^{\left(\eta-\frac{1}{2}\right)-1}(1-t)^{2 \theta-1}\left(1-\frac{t}{3}\right)^{2\left(\eta-\frac{1}{2}\right)-1}\left(1-\frac{t}{4}\right)^{\theta-1} \cos \left(t y\left(1-\frac{t}{3}\right)^{2}\right) d t \\
=\left(\frac{2}{3}\right)^{2(\eta+\theta)} \sqrt{\pi} \Gamma(\theta){ }_{1} \psi_{2}\left[\begin{array}{cc}
(\eta+\theta, 2) ; & {\left[\begin{array}{cc}
2 \\
\left(\eta+\theta-\frac{1}{2}, 2\right),\left(\frac{1}{2}, 1\right) ;
\end{array}\right]}
\end{array}\right.
\end{aligned}
$$

where $\Re(\eta)>0$ and $\Re(\theta)>0$.

Corollary 11. If we take $m=1, \mu=1, \lambda=0$ and $\nu=-1 / 2$ in (17) and then by 
using (7), we obtain:

$$
\begin{aligned}
& \int_{0}^{1} t^{\eta-1}(1-t)^{2\left(\theta-\frac{1}{2}\right)-1}\left(1-\frac{t}{3}\right)^{2 \eta-1}\left(1-\frac{t}{4}\right)^{\left(\theta-\frac{1}{2}\right)-1} \cos \left(y\left(1-\frac{t}{4}\right)\left(1-t^{2}\right)\right) d t \\
& =\left(\frac{2}{3}\right)^{2 \eta} \pi \Gamma(\eta)_{1} \psi_{3}\left[\begin{array}{cc}
\left(\theta-\frac{1}{2}, 4\right) ; & \frac{y^{4}}{64}
\end{array}\right] \\
& +\left(\frac{2}{3}\right)^{2 \eta}\left(\frac{\pi y^{2}}{16}\right) \Gamma(\eta)_{1} \psi_{3}\left[\begin{array}{cc}
\left(\theta+\frac{3}{2}, 4\right) ; & \frac{y^{4}}{64}
\end{array}\right]
\end{aligned}
$$

where $\Re(\eta)>0$ and $\Re(\theta)>0$.

Corollary 12. Further if we take $m=1, \mu=1, \lambda=0$ and $\nu=-1 / 2$ in (18) and then by using (7), we obtain:

$$
\begin{aligned}
& \int_{0}^{1} t^{\left(\eta-\frac{1}{2}\right)-1}(1-t)^{2 \theta-1}\left(1-\frac{t}{3}\right)^{2\left(\eta-\frac{1}{2}\right)-1}\left(1-\frac{t}{4}\right)^{\theta-1} \cos \left(t y\left(1-\frac{t}{3}\right)^{2}\right) d t \\
& =\left(\frac{2}{3}\right)^{2(\eta+\theta)} \pi \Gamma(\theta)_{1} \psi_{3}\left[\begin{array}{cc}
(\eta+\theta, 4) ; & \frac{y^{4}}{6561} \\
\left(\eta+\theta-\frac{1}{2}, 4\right),\left(\frac{1}{2}, 1\right),\left(\frac{1}{2}, 2\right) ; &
\end{array}\right] \\
& +\left(\frac{2}{3}\right)^{2(\eta+\theta)}\left(-\frac{8 \pi y^{2}}{81}\right) \Gamma(\theta)_{1} \psi_{3}\left[\begin{array}{cc}
(\eta+\theta+2,4) ; & y^{4} \\
\left(\eta+\theta+\frac{3}{2}, 4\right),\left(\frac{3}{2}, 1\right),\left(\frac{3}{2}, 2\right) ; & 6561
\end{array}\right],
\end{aligned}
$$

where $\Re(\eta)>0$ and $\Re(\theta)>0$.

Corollary 13. If we take $m=1$ in (11) and then by using (8), we obtain:

$$
\begin{aligned}
& \int_{0}^{1} t^{\eta-1}(1-t)^{2 \theta-1}\left(1-\frac{t}{3}\right)^{2 \eta-1}\left(1-\frac{t}{4}\right)^{\theta-1} \mathbb{J}_{\nu, \lambda}^{\mu}\left(y(1-t / 4)(1-t)^{2}\right) d t=\left(\frac{2}{3}\right)^{2 \eta}\left(\frac{y}{2}\right)^{\nu+2 \lambda} \Gamma(\eta) \\
& \times{ }_{2} \psi_{3}\left[\begin{array}{c}
(\theta+\nu+2 \lambda, 2),(1,1) ; \\
(\lambda+1,1),(\eta+\theta+\nu+2 \lambda, 2),(\nu+\lambda+1, \mu) ;
\end{array}\right]
\end{aligned}
$$

where $\Re(\eta)>0$ and $\Re(\theta)>0$. 
Corollary 14. Further if we take $m=1$ in (13) and then by using (8), we obtain:

$$
\begin{aligned}
& \int_{0}^{1} t^{\eta-1}(1-t)^{2 \theta-1}\left(1-\frac{t}{3}\right)^{2 \eta-1}\left(1-\frac{t}{4}\right)^{\theta-1} \mathbb{J}_{\nu, \lambda}^{\mu} t y(1-t / 3)^{2} d t \\
& =\left(\frac{2}{3}\right)^{2(\eta+\theta+2 \lambda)}\left(\frac{y}{2}\right)^{\nu+2 \lambda} \Gamma(\theta)_{2} \psi_{3}\left[\begin{array}{cc}
(\eta+\theta+2 \lambda, 2),(1,1) ; \\
(\lambda+1,1),(\eta+\theta+\nu+2 \lambda, 2),(1+\nu+\lambda, \mu) ;
\end{array}\right.
\end{aligned}
$$

where $\Re(\eta)>0$ and $\Re(\theta)>0$.

Corollary 15. If we take $m=1$ in (17) and then by using (8), we obtain:

$$
\begin{aligned}
& \int_{0}^{1} t^{\eta-1}(1-t)^{2 \theta-1}\left(1-\frac{t}{3}\right)^{2 \eta-1}\left(1-\frac{t}{4}\right)^{\theta-1} \mathbb{J}_{\nu, \lambda}^{\mu} y(1-t / 4)(1-t)^{2} d t \\
& =\left(\frac{2}{3}\right)^{2 \eta} \sqrt{\pi}\left(\frac{y}{2}\right)^{\nu+2 \lambda} \Gamma(\eta)_{2} \psi_{4}\left[\begin{array}{cc}
(1,2),(\theta+\nu+2 \lambda, 4) ; \\
(\lambda+1,2),(1 / 2,1),(\eta+\theta+\nu+2 \lambda, 4),(\nu+\lambda+1,2 \mu) ; & \frac{y^{4}}{64}
\end{array}\right] \\
& +\left(\frac{2}{3}\right)^{2 \eta} \sqrt{\frac{\pi}{2}\left(\frac{y}{2}\right)^{\nu+2 \lambda+2} \Gamma(\eta)} \\
& \times{ }_{2} \psi_{4}\left[\begin{array}{cc}
(2,2),(\theta+\nu+2 \lambda+2,4) ; & \frac{y^{4}}{64}
\end{array}\right]
\end{aligned}
$$

where $\Re(\eta)>0$ and $\Re(\theta)>0$.

Corollary 16. Further if we take $m=1$, in (18) and then by using (8), we obtain:

$$
\begin{aligned}
& \int_{0}^{1} t^{\eta-1}(1-t)^{2 \theta-1}\left(1-\frac{t}{3}\right)^{2 \eta-1}\left(1-\frac{t}{4}\right)^{\theta-1} \mathbb{J}_{\nu, \lambda}^{\mu} t y(1-t / 3)^{2} d t \\
& =\left(\frac{2}{3}\right)^{2(\eta+\theta+2 \lambda)} \sqrt{\pi}\left(\frac{y}{2}\right)^{\nu+2 \lambda} \Gamma(\theta)_{2} \psi_{4}\left[\begin{array}{c}
(1,2),(\eta+\theta+2 \lambda, 4) ; \\
(\lambda+1,2),\left(\frac{1}{2}, 1\right),(\eta+\theta+\nu+2 \lambda, 4),(\nu+\lambda+1,2 \mu) ;
\end{array}\right] \\
& +\left(\frac{2}{3}\right)^{2(\eta+\theta+2 \lambda)} \sqrt{\frac{\pi}{2}}\left(-\frac{16 y^{2}}{81}\right)\left(\frac{y}{2}\right)^{\theta+2 \lambda} \Gamma(\theta) \\
& \times{ }_{2} \psi_{4}\left[\begin{array}{cc}
(2,2),(\eta+\theta+2 \lambda+2,4) ; \\
(\lambda+2,2),\left(\frac{3}{2}, 1\right),(\eta+\theta+\nu+2 \lambda+2,4),(\nu+\lambda+\mu+1,2 \mu) ;
\end{array}\right]
\end{aligned}
$$

where $\Re(\eta)>0$ and $\Re(\theta)>0$.

Corollary 17. If we take $m=1, \mu=1$ and $\lambda=1 / 2$ in (11) and then by using (9), 
we obtain:

$$
\begin{aligned}
& \int_{0}^{1} t^{\eta-1}(1-t)^{2 \theta-1}\left(1-\frac{t}{3}\right)^{2 \eta-1}\left(1-\frac{t}{4}\right)^{\theta-1} \mathbb{H}_{\nu}\left(y(1-t / 4)(1-t)^{2}\right) d t \\
& =\left(\frac{2}{3}\right)^{2 \eta}\left(\frac{y}{2}\right)^{\nu+1} \Gamma(\eta)_{2} \psi_{3}\left[\begin{array}{cc}
(\theta+\nu+1,2),(1,1) ; \\
(\eta+\theta+\nu+1,2),(\nu+3 / 2,1),(3 / 2,1) ;
\end{array}\right],
\end{aligned}
$$

where $\Re(\eta)>0$ and $\Re(\theta)>0$.

Corollary 18. Further if we take $m=1, \mu=1$ and $\lambda=1 / 2$ in (13) and then by using (9), we obtain:

$$
\begin{aligned}
& \int_{0}^{1} t^{\eta-1}(1-t)^{2 \theta-1}\left(1-\frac{t}{3}\right)^{2 \eta-1}\left(1-\frac{t}{4}\right)^{\theta-1} \mathbb{H}_{\nu} t y(1-t / 3)^{2} d t=\left(\frac{2}{3}\right)^{2(\eta+\theta+1)}\left(\frac{y}{2}\right)^{\nu+1} \Gamma(\theta) \\
& \times{ }_{2} \psi_{3}\left[\begin{array}{c}
(\eta+\theta+1,2),(1,1) ; \\
(\eta+\theta+\nu+1,2),(\nu+3 / 2,1),(3 / 2,1) ;
\end{array}\right]
\end{aligned}
$$

where $\Re(\eta)>0$ and $\Re(\theta)>0$.

Corollary 19. If we take $m=1, \mu=1$ and $\lambda=1 / 2$ in (17) and then by using (9), we obtain:

$$
\begin{aligned}
& \int_{0}^{1} t^{\eta-1}(1-t)^{2 \theta-1}\left(1-\frac{t}{3}\right)^{2 \eta-1}\left(1-\frac{t}{4}\right)^{\theta-1} \mathbb{H}_{\nu} y(1-t / 4)(1-t)^{2} d t \\
& =\left(\frac{2}{3}\right)^{2 \eta} \sqrt{\pi}\left(\frac{y}{2}\right)^{\nu+1} \Gamma(\eta)_{2} \psi_{4}\left[\begin{array}{cc}
(1,2),(\theta+\nu+1,4) ; \\
(\eta+\theta+\nu+1,4),(\nu+3 / 2,2),(3 / 2,2),(1 / 2,1) ; & \left.\frac{y^{4}}{64}\right] \\
+\left(\frac{2}{3}\right)^{2 \eta} \sqrt{\frac{\pi}{2}}\left(\frac{y}{2}\right)^{\nu+3} \Gamma(\eta)_{2} \psi_{4}\left[\begin{array}{cc}
(\eta, 2),(\theta+\nu+3,4) ; \\
(\eta+\theta+\nu+3,4),(\nu+5 / 2,2),(5 / 2,2),(3 / 2,1) ;
\end{array}\right]
\end{array}\right.
\end{aligned}
$$

where $\Re(\eta)>0$ and $\Re(\theta)>0$.

Corollary 20. Further if we take $m=1, \mu=1$ and $\lambda=1 / 2$ in (18) and then by 
using (9), we obtain:

$$
\begin{aligned}
& \int_{0}^{1} t^{\eta-1}(1-t)^{2 \theta-1}\left(1-\frac{t}{3}\right)^{2 \eta-1}\left(1-\frac{t}{4}\right)^{\theta-1} \mathbb{H}_{\nu} t y(1-t / 3)^{2} d t \\
& =\left(\frac{2}{3}\right)^{2(\eta+\theta+1)} \sqrt{\pi}\left(\frac{y}{2}\right)^{\nu+1} \Gamma(\theta)_{2} \psi_{4}\left[\begin{array}{cc}
(1,2),(\eta+\theta+1,4) ; \\
(\eta+\theta+\nu+1,4),(\nu+3 / 2,2),(3 / 2,2),(1 / 2,1) ; & \frac{y^{4}}{6561}
\end{array}\right] \\
& +\left(\frac{2}{3}\right)^{2(\eta+\theta+1)} \sqrt{\frac{\pi}{2}}\left(-\frac{16 y^{2}}{81}\right)\left(\frac{y}{2}\right)^{\theta+1} \Gamma(\theta) \\
& \times{ }_{2} \psi_{4}\left[\begin{array}{c}
(2,2),(\eta+\theta+3,4) ; \\
(\eta+\theta+\nu+3,4),(\nu+5 / 2,2),(5 / 2,2),(3 / 2,1) ;
\end{array}\right]
\end{aligned}
$$

where $\Re(\eta)>0$ and $\Re(\theta)>0$.

\section{Reducibility of the Wright Hypergeometic Function}

Now we state two reduction formulas for the Wright hypergeometric function as follows:

$$
\begin{aligned}
& { }_{2} \psi_{m+2}\left[\begin{array}{c}
(\theta+\nu+2 \lambda, 2)(1,1) ; \\
(\lambda+1,1), \ldots(\lambda+1,1)(\eta+\theta+\nu+2 \lambda, 2)(\nu+\lambda+1, \mu) ;
\end{array} \quad-\frac{y^{2}}{4}\right] \\
& =\sqrt{\pi}_{2} \psi_{m+3}\left[\begin{array}{cc}
(\theta+\nu+2 \lambda, 4)(1,2) ; & y^{4} \\
(\lambda+1,2), \ldots(\lambda+1,2)(1 / 2,1)(\eta+\theta+\nu+2 \lambda, 4),(\nu+\lambda+1,2 \mu) ; & \frac{y^{6}}{64}
\end{array}\right]+\sqrt{\frac{\pi}{8}} y^{2} \\
& { }_{2} \psi_{m+3}\left[\begin{array}{cc}
(\theta+\nu+2 \lambda+2,4)(2,2) ; & y^{4} \\
(\lambda+2,2), \ldots(\lambda+2,2)(\eta+\theta+\nu+2 \lambda+2,4)\left(\frac{3}{2}, 1\right)(\mu+\nu+\lambda+1,2 \mu) ; & \frac{y^{4}}{64}
\end{array}\right] \\
& { }_{2} \psi_{m+2}\left[\begin{array}{cc}
(\eta+\theta+2 \lambda, 2)(1,1) ; & -4 y^{2} \\
(\lambda+1,1), \ldots(\lambda+1,1),(\eta+\theta+\nu+2 \lambda, 2),(1+\nu+\lambda, \mu) ; & \left.\frac{81}{8}\right]
\end{array}\right. \\
& =\sqrt{\pi}{ }_{2} \psi_{m+3}\left[\begin{array}{cc}
(\eta+\theta+2 \lambda, 4)(1,2) ; & y^{4} \\
(\lambda+1,2), \ldots(\lambda+1,2)(1 / 2,1),(\eta+\theta+\nu+2 \lambda, 4)(\nu+\lambda+1,2 \mu) ; & 6561
\end{array}\right] \\
& +\sqrt{\pi}\left(-\frac{4 y^{2}}{81}\right) \\
& \times{ }_{2} \psi_{m+3}\left[\begin{array}{cc}
(\eta+\theta+2 \lambda+2,4)(2,2) ; & y^{4} \\
(\lambda+2,2), \ldots(\lambda+2,2)(3 / 2,1)(\eta+\theta+\nu+2 \lambda+2,4)(\nu+\lambda+\mu+1,2 \mu) ; & 6561
\end{array}\right]
\end{aligned}
$$

By comparing (11) and (17),results (35) can be established and by comparing (14) and (18),result (36) can be established.

\section{Declaration}

The preprint of the paper is available at "https://www.preprints.org/manuscript/201802.0155/v1" 
S. Haq, K.S. Nisar, A.H. Khan - Some new results associated with ...

\section{REFERENCES}

[1] K.N. Bhowmick, Some relation between generalized Struve function and hypergeometric function, Vijnana Parishad Anusandhan Patrika,5 93-99.

[2] J. Choi and P. Agarwal, Certain unified integrals associated with Bessel functions, Bound. Value Probl. 95 (2013).

[3] J. Choi, P. Agarwal, S. Mathur and S.D. Purohit, Certain new integral formulas involving the generalized Bessel function, Bull. Korean Math. Soc.(2014) no. 4, 995-1003.

[4] J. Choi, K.S. Nisar; Certain families of integral formulas involving Struve function. Boletim da Sociedade Paranaense de Matematica, 37(3), 2019, 27-35

[5] K.B. Kachhia and J.C. Prajapati, On generalized fractional kinetic equations involving generalized Lommel-Wright functions, Alexandria Engineering Journal (elsevier) (2016)55, 2953-2957.

[6] N.U. Khan, S.W. Khan,M.Ghayasuddin, Some new results associated with the Bessel struve kernel function, Acta Universitatis Apulensis,no.48(2016),pp.89-101.

[7] J.P. Konovska. Theorems on the convergence of series in generalized Lommel-Wright functions. Fractional Calculus and Applied Analysis, Vol.10, no.1(2007).

[8] J.L. Lavoie and G.Trottier, On the sum of certain Appell's series, Ganita, (1) 20(1969), $31-32$.

[9] K.S. Nisar, G. Rahman, A. Ghaffar, S.A. Mubeen, New class of integrals involving extended Mittag-Leffler function Journal of Fractional Calculus and Applications 9 (1), 222-231, (2018)

[10] K.S. Nisar, S.R. Mondal, Certain unified integral formulas involving the generalized modified $k$-Bessel function of first kind, Communication of Korean Mathematical Society, 32 (1), 2017. , pp. 47-53

[11] K.S. Nisar, W.A. Khan, Beta type integral operator associated with Wright generalized Bessel function. Acta Mathematica Universitatis Comenianae, 871 117-125 2018

[12] K.S. Nisar, D. Baleanu and M.M. Al Qurashi, Fractional calculus and application of generalized Struve function, Springer Plus (2016)5:910,DOI 10.1186/s40064-016-2560-3.

[13] E.D. Rainville, Special Functions, Macmillan, New York, 1960.

[14] R.P. Singh, Some integral representation of generalized Struve's functions, Math.Ed (Siwan), 22(3) (1988), 91-94.

[15] H.M. Srivastava, and H.L. Manocha, A treatise on generating functions, John Wily and Sons (Halsted Press, New York,Ellis Horwood,Chichester).

Sirazul Haq

Department of Applied Mathematics, Faculty of Engineering and Technology,

Aligarh Muslim University,

Aligarh-202002, UP, India,

email: asirajulhaq007@gmail.com

Kottakkaran Sooppy Nisar

Department of Mathematics, College of Arts and Science,

Prince Sattam bin Abdulaziz University, 
S. Haq, K.S. Nisar, A.H. Khan - Some new results associated with ...

Wadi Aldawaser, Riyadh region 11991, Saudi Arabia

email: n.sooppy@psau.edu.sa, ksnisar1@gmail.com

Abdul Hakim Khan

Department of Applied Mathematics, Faculty of Engineering and Technology, Aligarh Muslim University, Aligarh-202002, UP, India, email: ahkhan.amu@gmail.com 